\title{
PARTICLE IMAGE VELOCIMETRY MEASUREMENTS OF STRATIFIED GAS-LIQUID FLOW IN HORIZONTAL AND INCLINED PIPES
}

\author{
S. VESTØL, W.A.S. KUMARA \& M.C. MELAAEN \\ University College of Southeast Norway, Porsgrunn, Norway.
}

\begin{abstract}
The main objectives of this work is to produce detailed velocity profile measurements over a range of operating conditions of two phase gas/liquid flow with low liquid fractions in horizontal and inclined pipes. The experiments are performed in a $15 \mathrm{~m}$ long stainless steel pipe section with internal diameter $56 \mathrm{~mm}$ at room temperature and atmospheric outlet pressure. Exxsol D60 oil (viscosity $1.30 \mathrm{mPa} \mathrm{s}$, density $793 \mathrm{~kg} / \mathrm{m}^{3}$ ), water (viscosity $0.89 \mathrm{mPa} \mathrm{s}$, density $999 \mathrm{~kg} / \mathrm{m}^{3}$ ) and air (viscosity $0.018 \mathrm{mPa} \mathrm{s}$, density $1.22 \mathrm{~kg} / \mathrm{m}^{3}$ ) are used as test fluids. The pipe inclination is changed in the range from $5^{\circ}$ upward to $5^{\circ}$ downward. The measurements are made at mixture velocity, $5 \mathrm{~m} / \mathrm{s}$ for different inlet liquid fractions. The cross-sectional distribution of phase fractions is measured using a traversable single-beam gamma densitometer. The particle image velocimetry (PIV) is utilized in order to obtain non-invasive instantaneous velocity measurements of the flow field. Based on the instantaneous local velocities, mean velocities, root mean squared velocities and Reynolds stresses are calculated. The measured mean velocity and turbulence profiles show a strong dependency with pipe inclination. The present measurements show that PIV can be successfully used as a practical measurement technique for multiphase flow applications with potential to become even more powerful in the near future as digital camera technology progresses.

Keywords: gamma densitometer, local velocity, mean velocity, mixture velocity, particle image velocimetry, root mean squared velocity
\end{abstract}

\section{INTRODUCTION}

Gas/liquid flows occur in many industrial processes including chemical reactors, thermal hydraulic circuits in nuclear power reactors and pipelines and equipment in the oil and gas industry. In all these applications, the flow physics and interaction of phases with each other have to be understood. Yet, many important fluid dynamical aspects of these two-phase flow applications are still poorly understood. This explains to some extent the considerable effort made by various researchers to develop sophisticated Computational Fluid Dynamics (CFD) models for gas/liquid flow applications especially in offshore transportation pipelines. In order to accurately assess the validity of these CFD models, quantitative experimental data are required on the instantaneous flow phenomena and on the coherent structures dominating the flow.

The rapid developments within lasers and camera technology opened the possibility for qualifying (flow visualization) and later quantifying complex flow fields. The development of particle image velocimetry (PIV) has become one of the most popular non-intrusive techniques for flow measurements in numerous multiphase flow applications. In contrast to the single-point Laser Doppler Anemometry (LDA) measurements, PIV represents an instantaneous whole field measurement technique, which makes it possible to detect the spatial flow structure providing a direct indication of the interphase coupling in multiphase flows. The principles of PIV have been covered in many papers including Adrian [1], Hassan et al. [2], Westerweel [3] and Jensen [4]. The PIV has been used in the measurement of single-phase flow in many different operational modes since the late 1970s. However, it has only recently been applied to multiphase flow systems. Hassan et al. [2] applied PIV technique for the 
simultaneous, two-dimensional velocity measurements of both gas and liquid phases of dispersed air bubble mixing flow in a rectangular vessel. They were able to use the velocity information obtained by the PIV system to determine the liquid streamlines and vorticity fields. Chen et al. [5] have applied the PIV technique to simultaneously measure, the instantaneous full-field properties of the velocity components in gas/liquid/solid fluidization system. Birvalski et al. [6] used planar PIV coupled with a profile detection technique in order to investigate stratified flow pattern of air and water in a $50 \mathrm{~mm}$ diameter pipe. Detailed velocity measurements were presented for laminar, transitional, and turbulent liquid layers in air/water flows. Ayati et al. [7] presented detailed velocity measurements in both gas and liquid phases for horizontal air/water flow using PIV. They have combined PIV and conductance probe technique to obtain instantaneous velocity and turbulence measurements and wave statistics such as, wave amplitudes and speeds as well as power spectral densities.

The objective of this study is to apply PIV to the full-field non-intrusive analysis of gas/ liquid flow in horizontal and slightly inclined pipes. The mixture velocity, liquid fractions and pipe inclinations used in the experiments are in a range that is commonly used in transportation of unprocessed gas. The local mean axial velocities and root mean square (RMS) velocities are presented. The cross-sectional phase distribution of the flow is measured using a single-beam gamma densitometer.

\section{EXPERIMENTAL SET-UP}

2.1 Multiphase flow loop

A simplified flow sheet of the experimental rig is shown in Fig. 1, which has been presented in detail by Kumara et al. [8]. Three fluid phases are available in the multiphase flow

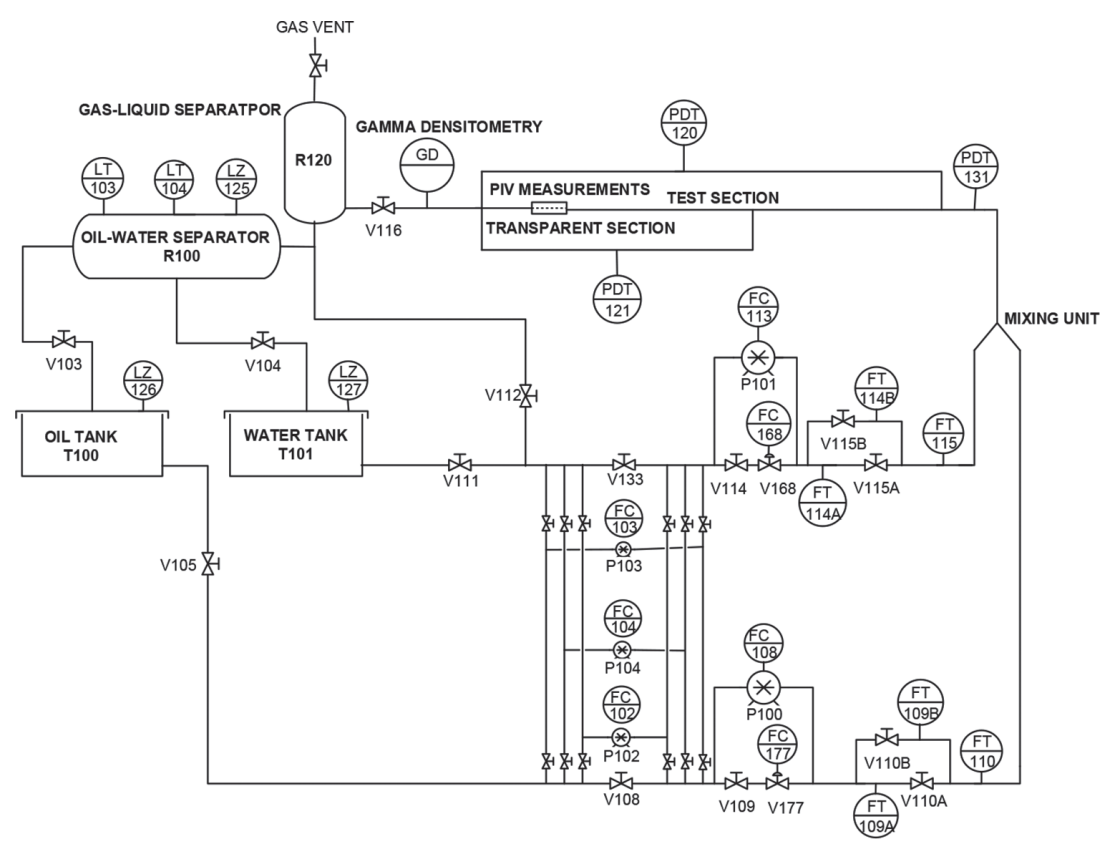

Figure 1: Simplified flow sheet of the test rig. 
Table 1: Physical properties of test fluids at atmospheric conditions.

\begin{tabular}{llll}
\hline & Gas & Oil & Water \\
\hline Fluid & Air & Exxsol D60 & Tap water \\
Density at $15^{\circ} \mathrm{C}$ & $1.22 \mathrm{~kg} / \mathrm{m} 3$ & $793 \mathrm{~kg} / \mathrm{m} 3$ & $999 \mathrm{~kg} / \mathrm{m} 3$ \\
Viscosity at $25^{\circ} \mathrm{C}$ & $0.018 \mathrm{mPa} \cdot \mathrm{s}$ & $1.3 \mathrm{mPa} \cdot \mathrm{s}$ & $0.89 \mathrm{mPa} \cdot \mathrm{s}$ \\
Surface tension at $25^{\circ} \mathrm{C}$ & & $25.9 \mathrm{mN} / \mathrm{m}$ & $72.0 \mathrm{mN} / \mathrm{m}$ \\
\hline
\end{tabular}

facility: gas, oil and water. Oil and water are stored in separate tanks, T100 and T101, respectively. Seven different pumps (P100, P101, P102, P103, P104, P105 and P106) are available to circulate the liquid phases through the test system. Air is supplied from the central pressurized air system in the building, and the air flow rate is controlled with a pneumatic control valve (V131). The three fluid phases are mixed at the inlet of the test section which has been designed to initiate separated flow. The test section is made of steel, except for two short plastic sections. A $0.8 \mathrm{~m}$ long transparent acrylic section is used to allow visual observations of the flow while $0.4 \mathrm{~m}$ long polypropylene section is used to allow measurements with the gamma densitometer. The length of the test pipe section is $15 \mathrm{~m}$ and inner diameter is $56.3 \mathrm{~mm}$. A gas/liquid separator (R120) is located immediately downstream the test section. The liquid outlet of the gas/liquid separator is connected to an oil/water separator (R100) located at a lower elevation where the oil and water phases are separated and returned to their respective storage tanks. Table 1 lists some important physical properties of the test fluids under atmospheric conditions. A controller based on LabView ${ }^{\circledR}$ is used for setting gas and liquid inflow rates and selecting appropriate pumps and flow meters. The flow rates are controlled by an automatic control loop, which uses the flow meter readings to adjust the pump speeds and valve openings.

\subsection{PIV set-up}

The PIV set-up consists of a charge coupled device (CCD) camera with 1260x1024 pixel resolution (1.3Mpx), a high-power double-pulsed Litron laser and signal processing system as shown in Fig. 2. Two laser pulses with a short time delay are required for PIV measurements. For this purpose, a Litron laser type NANO-L-50-100 PIV is used. It includes two laser cavities to allow double pulses. The shortest delay time used in the experiments is $100 \mu \mathrm{s}$. Both laser cavities have a maximum frequency of $100 \mathrm{~Hz}$.

To allow single exposure double image PIV, a high-speed camera that can record image pairs with a short time delay is needed. A Nanosense Mk1 camera is used for this purpose. This camera has a maximum frame rate of $630 \mathrm{~Hz}$ at maximum resolution, and it can be operated in double exposure mode. The camera is fitted with an AF Micro-Nikkor 60mm f/2.8D lens and a green filter that filtered all incoming light of different wavelength from the laser light. The camera resolution is $1280 \times 1024$ pixels. The software used for PIV data acquisitions and analyses is FlowManager version 4.71 from Dantec Dynamics. The timing of the laser and the camera is controlled and synchronized through FlowManager. An adaptive correlation routine available in FlowManager software is used to find the velocity vectors from an image pair. 


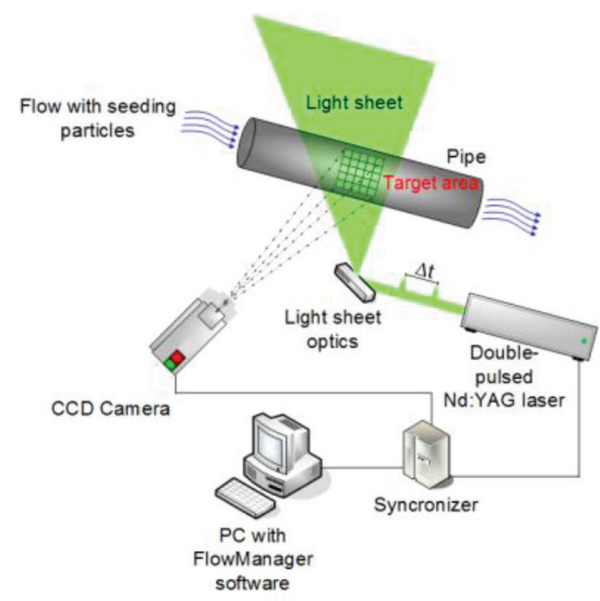

Figure 2: PIV set-up used in the experiments.

\subsection{Seeding particles}

PIV measures the velocity based on an average particle displacement between two corresponding interrogation areas in two images of the flow. This approach requires tracer particles to be present in the flow. The liquid phases are seeded with solid polyamide tracer particles with an average diameter of $20 \mu \mathrm{m}$ and the gas phase is seeded with droplets of Shell Ondina oil type 917 with average diameter of $2 \mu \mathrm{m}$.

\subsection{Investigated flow conditions}

The mixture velocity, $\mathrm{U}_{\mathrm{m}}$, of gas/liquid flow is defined as follows.

$$
U_{m}=\frac{Q_{g}+Q_{l}}{A}(1)
$$

where $Q_{g}$ and $Q_{l}$ are the inlet volumetric flow rates of gas and liquid, respectively, and $A$ is the pipe cross-sectional area. In the present work, mixture velocity is kept at $5 \mathrm{~m} / \mathrm{s}$. The inlet liquid fraction, $\lambda_{l}$, is defined as

$$
\lambda_{l}=\frac{Q_{l}}{Q_{g}+Q_{l}}(2)
$$

The experiments are performed at five different inlet liquid fractions of $0.0010,0.0025$, $0.0050,0.0075$ and 0.0100 and at pipe inclinations of $-5^{\circ},-1^{\circ}, 0,+1^{\circ}$ and $+5^{\circ}$.

\section{RESULTS}

3.1 Horizontal air/water flows with mixture velocity $5 \mathrm{~m} / \mathrm{s}$ and liquid fraction 0.0100

Figure 3(a) shows the average velocity profile in axial direction in the liquid phase for a horizontal air/water flow with mixture velocity $5 \mathrm{~m} / \mathrm{s}$ and liquid fraction 0.0100 . The dotted line 
near the top of the figure is the interface position taken from the gamma measurements. The figure shows that the gradient is large close to the wall and close to the interface, and there is almost no gradient in the middle of the liquid phase. This is in agreement with the results presented by Olive et al. [9] and Sutharshan et al. [10]. This result can be explained with an analogy to turbulent flow between a stagnant and a moving wall, where the interface is looked upon as a moving wall. The no-slip condition gives that the liquid velocity will be zero at the stagnant wall. The gradient close to the stagnant wall will be large, but decrease with distance from the wall. At the moving wall (the interface), the velocity will be the same as the moving wall velocity (interface velocity). The gradient will be large close to the moving wall (interface), but decrease with distance away from the interface. Far away from the walls, there will be no velocity gradient in turbulent flow. The measurement results agree well with this analogy.

Figure $3 b$ illustrates axial turbulence profiles, calculated as root mean square (RMS) values. The figure shows that the turbulence is high close to the wall and to the interface, and lower in the middle of the liquid phase. The resolution of PIV measurements is limited by the camera resolution. For practical applications with good quality seeding and proper camera settings like used in the experiments, sub-pixel interpolation can give accuracy down to about 0.1 pixel (FlowManager software user guide, 2000). For this flow, 0.1 pixel corresponds to about 0.003 $\mathrm{m} / \mathrm{s}$ for the measurements in the liquid phase and $0.006 \mathrm{~m} / \mathrm{s}$ for the measurements in the gas phase. The turbulence scales in this flow are too small compared to the resolution of the PIV measurements to ensure very accurate measurements.

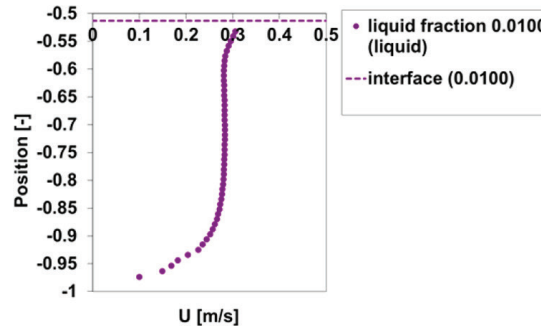

(a)

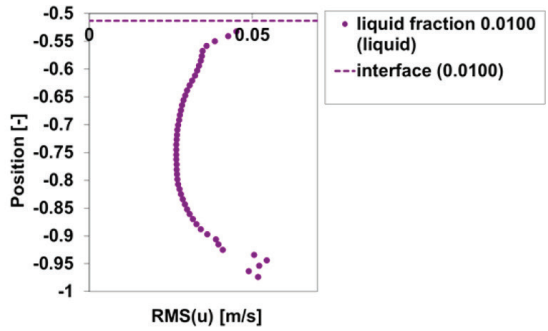

(b)

Figure 3: Axial velocity profile in liquid phase for a horizontal air/water flow with mixture velocity $5 \mathrm{~m} / \mathrm{s}$ and liquid fraction 0.0100 .

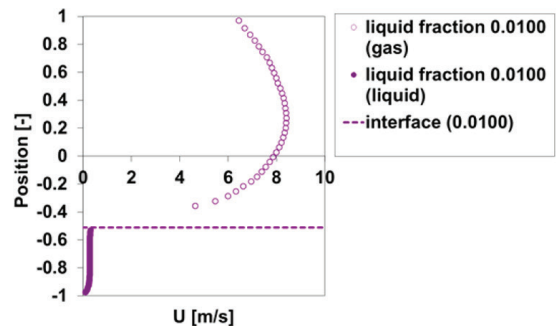

(a)



(b)

Figure 4: Axial velocity profile in gas and liquid phase for a horizontal air/water flow with mixture velocity $5 \mathrm{~m} / \mathrm{s}$ and liquid fraction 0.0100 . 
The axial velocity profile in both the gas and the liquid phase for a horizontal air/water flow with mixture velocity $5 \mathrm{~m} / \mathrm{s}$ and liquid fraction 0.0100 is shown in Fig. 4a. The figure shows that the velocity in the gas phase is a lot higher than in the liquid phase. The maximum velocity is close to halfway between the interface and the upper wall. The velocity profile can be explained with an analogy to turbulent flow between a smooth wall and a rough wall, where the interface is looked upon as a rough wall because of the interfacial waves. The noslip condition gives that the gas velocity will be zero at the smooth pipe wall and equal to the liquid velocity at the interface. The liquid is moving very slowly compared to the gas, so the rough interface will give more resistance than the smooth pipe wall. Then the velocity profile is expected to be skewed so the maximum value appears closer to the smooth wall as presented by Newton et al. [11]. This is not the case in the present results. This could be caused by secondary flows, which are known to skews the maximum downwards again see Meknassi et al. [12]. Presence of secondary flow could indicate that the flow is not fully developed. Figure $4 \mathrm{~b}$ shows the vertical velocity measurements for this flow. The figure indicates that secondary flow is present in the gas phase as downward flow in the center plane where the measurements are taken.

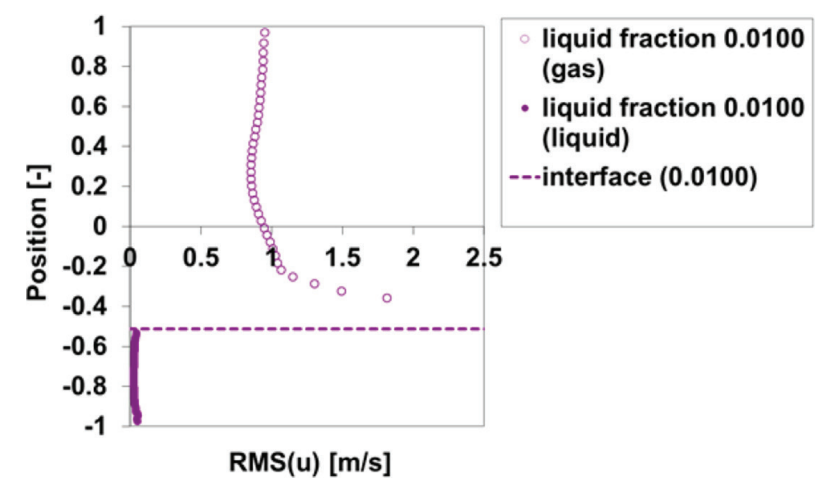

Figure 5: Axial turbulence profile in gas and liquid phase for a horizontal air/water flow with mixture velocity $5 \mathrm{~m} / \mathrm{s}$ and liquid fraction 0.0100 .



(a)

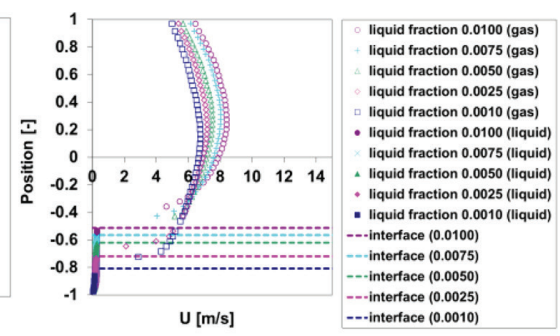

(b)

Figure 6: Axial velocity profiles in liquid phase for horizontal air/water flows with mixture velocity $5 \mathrm{~m} / \mathrm{s}$ and different liquid fractions. 
Figure 5 shows the turbulence profile in axial direction in a horizontal air/water flow with mixture velocity $5 \mathrm{~m} / \mathrm{s}$ and liquid fraction 0.0100 . The figure shows that the gas phase is lot more turbulent than the liquid phase, and the turbulence is largest close to the interface.

\subsection{Horizontal air/water flows with mixture velocity $5 \mathrm{~m} / \mathrm{s}$ and different liquid fractions}

Time averaged velocity profiles in the liquid phase for horizontal air/water flows with mixture velocity $5 \mathrm{~m} / \mathrm{s}$ and different liquid fractions are compared in Fig. 6a. The interface positions measured with a traversable gamma densitometer are indicated with the dotted lines in the figure. The liquid velocity increases with increasing liquid fraction. The liquid velocity gradient is large close to the bottom wall and close to the interface. In the middle of the liquid phase, the velocity is almost constant. This is in agreement with the predictions presented by Newton et al. [11] and Meknassi et al. [12]. Figure 6b shows the axial velocity profiles in both the gas and the liquid phases. The liquid velocity is very small compared to the gas velocity. The gas velocity profiles have a maximum value around halfway between the interface and the upper wall, and the maximum value increase with increasing liquid fraction because higher liquid fraction gives a smaller gas flow area. Except for close to the upper wall and close to the interface, the gas velocity gradient in the area above the maximum value is smaller than in the area below the maximum value. For the smallest liquid fractions, there are only small differences in the gas velocity gradient above and below the maximum value, but the difference increases with increasing liquid fraction.

Turbulence RMS profiles in axial direction are shown in Figs. 7a and b for liquid and gas phases, respectively. The measurements on flows with different liquid fractions but the same pipe inclination and mixture velocity are always done immediately after each other in one single experimental series. This appears to give consistent results within the measurement series, but it was difficult to later reproduce the absolute values of the turbulence measurements. When re-doing some measurements, deviations in the turbulence measurements between the first and second measurement series of up to about $30 \%$ have been observed. This may be due to slightly different alignment of the laser sheet or the camera, which will give small differences in the sub-pixel interpolation. Since the turbulence scales are small, the resolution of the PIV measurements is poor for the turbulence measurements, and small differences in the sub-pixel interpolation may cause significant differences in the results. However, the trends are easily reproducible. All the RMS profiles in axial direction in the liquid phase show a minimum value around the middle of the liquid phase and have largest

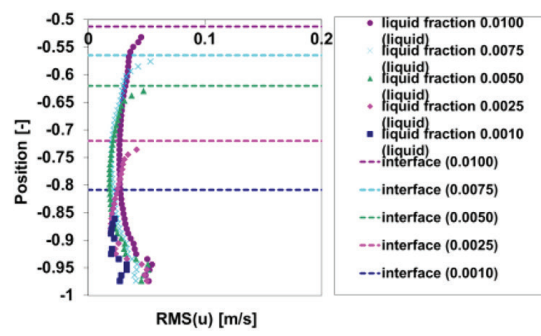

(a)

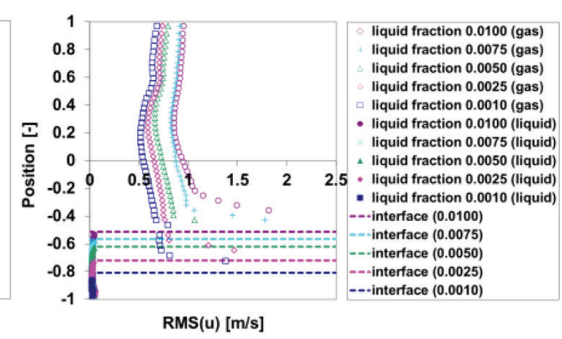

(b)

Figure 7: Axial turbulence profiles in liquid phase for horizontal air/water flows with mixture velocity $5 \mathrm{~m} / \mathrm{s}$ and different liquid fractions. 
gradients near the walls and the interface. The RMS profiles show much higher turbulence in the gas phase than in the liquid phase. The RMS profiles in the gas phase also have a minimum value around halfway between the wall and the interface and have largest gradients near the walls and the interface. The minimum RMS values in axial direction tend to increase with increasing liquid fraction both for the liquid phase and for the gas phase.

\subsection{Horizontal air/oil flows with mixture velocity $5 \mathrm{~m} / \mathrm{s}$ and different liquid fractions}

Time averaged velocity profiles for horizontal air/oil flows with mixture velocity $5 \mathrm{~m} / \mathrm{s}$ and different liquid fractions are compared in Figs. $8 \mathrm{a}$ and $\mathrm{b}$. The velocity profiles are very similar to the corresponding air/water flows described in Section 3.2, except for the liquid velocity profiles for liquid fractions 0.0010 and 0.0025 . The liquid film is slightly thinner for the air/ oil flows compared to the air/water flows. Because of this, the gas velocities in the air/oil flows are slightly lower and the liquid velocities are slightly higher compared to the similar measurements of air/water flows. The velocity gradient in the middle of the liquid phase in the air/oil flows is larger than in the corresponding air/water flows.

The velocity profiles in the liquid phase for the flow cases with liquid fraction 0.0010 and 0.0025 showed in Fig. 8a are fundamentally different from the rest. Those two flow cases give almost linear velocity profiles all the way from the wall to the interface. The explanation for this is that it is laminar flow in the liquid film in those two flow cases. According to Valle [13], a Reynolds number based on an equivalent hydraulic diameter can be used to predict the transition between laminar and turbulent flow in stratified two-phase flow. Reynolds numbers below 2300 based on the hydraulic diameter should give laminar flow. The estimated Reynolds number for the horizontal air/oil flows with mixture velocity $5 \mathrm{~m} / \mathrm{s}$ and liquid fraction 0.0010 and 0.0025 are 923 and 1776, respectively, and should be laminar in the liquid phase, while the flow with liquid fraction 0.0050 has a Reynolds number of 2928 and should be in the transition to turbulent flow. The transition in the velocity profiles from the PIV measurements agrees well with this theory. Visual observations indicate that this transition also coincides with the flow pattern transition between stratified smooth and stratified wavy flow, since the two lowest liquid fractions give stratified smooth and the other flows are stratified wavy without entrainment. However, this theory does not agree that well for air/water flows. For the horizontal air/ water flow case with mixture velocity $5 \mathrm{~m} / \mathrm{s}$ and liquid fraction 0.0010 , the PIV measurements give a velocity profile similar to those typical for turbulent flows, while the theory says it should be laminar in the liquid phase $(\operatorname{Re}=1390)$, see Fig. 6a. Visual observations show that this flow alternated between the flow patterns stratified smooth and stratified wavy.

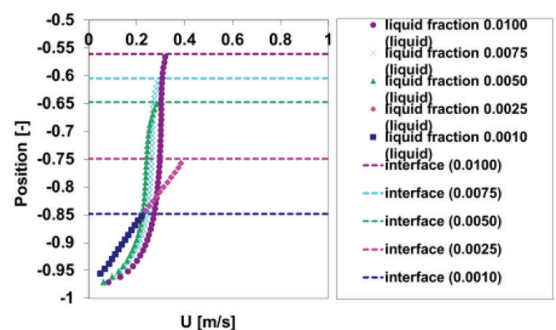

(a)

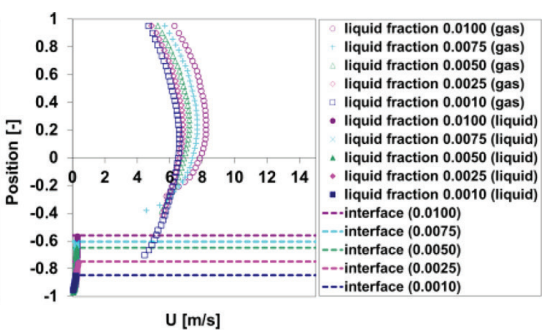

(b)

Figure 8: Axial velocity profiles in liquid phase for horizontal air/oil flows with mixture velocity $5 \mathrm{~m} / \mathrm{s}$ and different liquid fractions. 


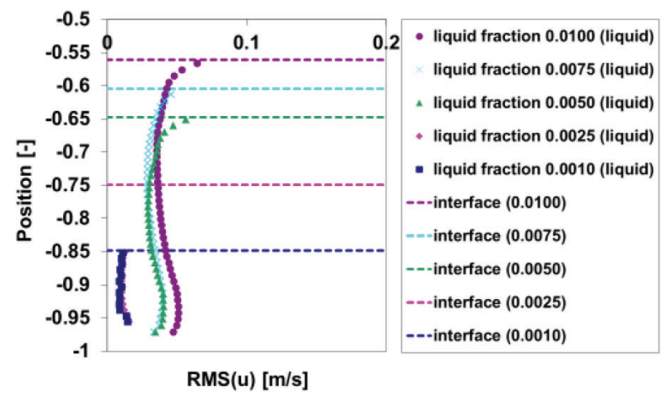

Figure 9: Axial turbulence profiles in liquid phase for horizontal air/oil flows with mixture velocity $5 \mathrm{~m} / \mathrm{s}$ and different liquid fractions.

The turbulence RMS profiles in axial direction for the liquid phase are shown in Fig. 9. It can be seen that the measured turbulence in the liquid phase for the flows with liquid fraction 0.0010 and 0.0025 are almost flat and significantly lower than the rest, like expected for laminar flows. In general, the turbulence must be zero in laminar flow, but measurement noise will make the measurements show some turbulence. This is a good indication of the accuracy of the measured absolute RMS values. The true RMS values for the laminar flows are known to be zero while the maximum measured RMS value for the laminar flows is $0.016 \mathrm{~m} / \mathrm{s}$, so the measurement noise in these RMS measurements are around $0.016 \mathrm{~m} / \mathrm{s}$.

\subsection{Air/water flows with mixture velocity $5 \mathrm{~m} / \mathrm{s}$, liquid fraction 0.0100 and different pipe} inclinations

Figure 10a and b compare axial velocity measurements for air/water flows with mixture velocity $5 \mathrm{~m} / \mathrm{s}$ and liquid fraction 0.0100 for different pipe inclination angles. The flows with upwards inclinations are not shown since they give slug flow. Increasing downward inclination give larger liquid velocity, but the liquid velocity is very small compared to the gas velocity. The shape of the axial velocity profiles in the gas phase is close to what is observed for the horizontal flow measurements. Increased downward inclination gives a thinner liquid layer. This makes crosssectional area for the gas flow larger, and therefore, the gas velocities are smaller for increasing downwards inclinations. The differences in the gas velocities between horizontal and $-1^{\circ}$ inclination are much larger than the differences in the gas velocities between $-1^{\circ}$ and $-5^{\circ}$ inclinations.



(a)



(b)

Figure 10: Axial velocity profiles in liquid phase for air/water flows with mixture velocity $5 \mathrm{~m} / \mathrm{s}$, liquid fraction 0.0100 and different pipe inclinations. 


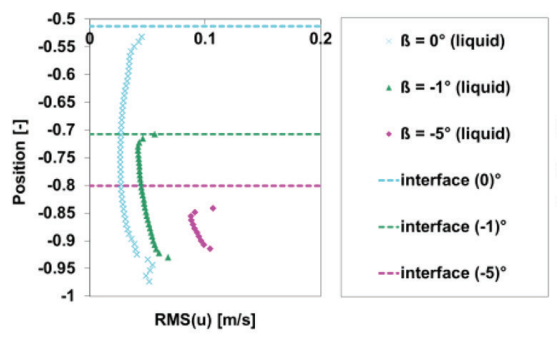

(a)



(b)

Figure 11: Axial turbulence profiles in liquid phase for air/water flows with mixture velocity $5 \mathrm{~m} / \mathrm{s}$, liquid fraction 0.0100 and different pipe inclinations.

Figure 11a shows axial turbulence profiles in the liquid phase based on the velocity measurements from Fig. 10a. The turbulence profiles follow the same trends observed in horizontal air/water flows. Figure 11b gives the turbulence profiles in both the gas and the liquid phases. The turbulence in the gas phase is much higher than in the liquid phase because the gas velocities are much higher than the liquid velocities. The turbulence in the gas phase increases close to the interface like in the horizontal air/water flow measurements.

\subsection{Air/oil flows with mixture velocity $5 \mathrm{~m} / \mathrm{s}$, liquid fraction 0.0100 and different pipe inclinations}

Axial velocity measurements for air/oil flows with mixture velocity $5 \mathrm{~m} / \mathrm{s}$, liquid fraction 0.0100 and different pipe inclinations are given in Figs 12a and b. Upward inclinations are not shown because they give slug flow. At pipe inclination, $-5^{\circ}$ the PIV measurements failed in large parts of the liquid phase because of optical disturbances from reflections caused by the short distance between the interface and the bottom wall. The thickness of the liquid layer for the $-5^{\circ}$ case is higher than in the same air/water case, see Fig. 10a, and the liquid velocity profile gives lower velocities in the air/oil case compared to the air/water case. Horizontal flow and flow with pipe inclination $-1^{\circ}$ give liquid velocity profiles that are very similar to the corresponding velocity profiles observed in air/water flows.

The axial turbulence RMS profiles are given in Figs 13a and b for air/oil flows with mixture velocity $5 \mathrm{~m} / \mathrm{s}$, liquid fraction 0.0100 and different pipe inclinations. Considering the accuracy of turbulence profiles acquired from PIV measurements, the results are very similar to the corresponding air/water cases presented in Figs 11a and b.

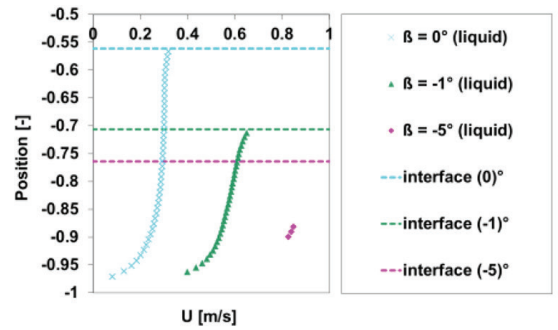

(a)

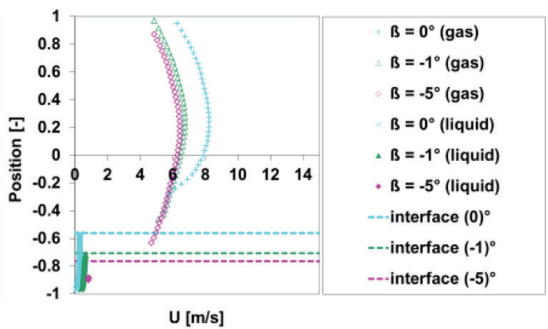

(b)

Figure 12: Axial velocity profiles in liquid phase for air/oil flows with mixture velocity $5 \mathrm{~m} / \mathrm{s}$, liquid fraction 0.0100 and different pipe inclinations. 


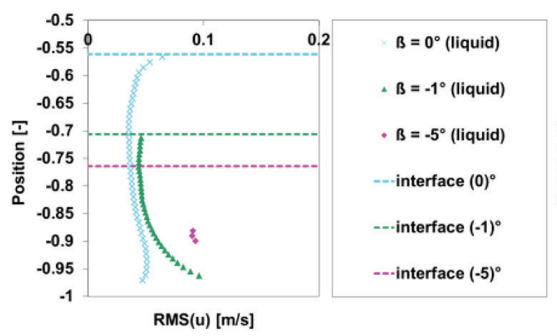

(a)



(b)

Figure 13: Axial turbulence profiles in liquid phase for air/oil flows with mixture velocity $5 \mathrm{~m} / \mathrm{s}$, liquid fraction 0.0100 and different pipe inclinations.

\section{CONCLUSIONS}

Detailed measurements of gas/liquid flows for a range of flow conditions are presented. PIV is used to measure instantaneous velocity profiles both in gas and liquid phases, and turbulence profiles are estimated based on the velocity measurements. The interface position is measured using single beam gamma densitometry and presented together with PIV measurements. The measurements covers air/water flows and air/oil flows with mixture velocity $5 \mathrm{~m} / \mathrm{s}$ and liquid fractions $0.0010,0.0025,0.0050,0.0075$ and 0.0100 , and pipe inclinations $-5^{\circ},-1^{\circ}$ $, 0^{\circ},+1^{\circ}$ and $+5^{\circ}$.

In general, higher axial velocities are observed in the gas phase. Higher velocity gradients are observed close to the pipe wall and interface. Based on the horizontal gas-liquid flow measurements, the maximum velocity is located close to halfway between the interface and the upper wall. This could be caused by secondary flows similar to the results presented by Meknassi et al. [12]. The current measurements show the presence of secondary flows in the gas phase and it indicates that the flow is not fully developed.

The water velocity increases with increasing liquid fraction in horizontal air-water flows at mixture velocity $5 \mathrm{~m} / \mathrm{s}$. The liquid velocity gradient is large close to the bottom wall and close to the interface. In the middle of the liquid phase, the velocity is almost constant. The maximum gas velocity increases with increasing liquid fraction because higher liquid fraction gives a smaller gas flow area. RMS velocity profiles shows significantly higher turbulence levels in the gas phase compared to the water phase, and the turbulence is largest close to the interface. Laminar flow is observed in the liquid layer in horizontal air/oil flows at mixture velocity $5 \mathrm{~m} / \mathrm{s}$ and at low liquid fractions 0.0100 and 0.0025 . Visual observations indicate that this transition also coincides with the flow pattern transition between stratified smooth and stratified wavy flow, since the two lowest liquid fractions give stratified smooth and the other flows are stratified wavy without entrainment. However, horizontal air/water flows with mixture velocity $5 \mathrm{~m} / \mathrm{s}$ and liquid fraction 0.0010 and 0.0025 produce velocity profile similar to typical turbulent flows.

Higher liquid velocities are observed in downwardly inclined flows. Increased downward inclination gives a thinner liquid layer. This makes cross-sectional area for the gas flow larger, and therefore, the gas velocities are smaller for increasing downwards inclinations. The differences in the gas velocities between horizontal and $-1^{\circ}$ inclination are much larger than the differences in the gas velocities between $-1^{\circ}$ and $-5^{\circ}$ inclinations. PIV measurements failed in upwardly inclined pipes due to optical disturbances caused by slug flow. 


\section{REFERENCES}

[1] Adrian, R.J., Particle imaging techniques for experimental fluid mechanics. Annual Review of Fluid Mechanics, 23, pp. 261-304, 1991. https://doi.org/10.1146/annurev.fluid.23.1.261

[2] Hassan, Y.A., Blanchat, T.K., Seeley, C.H. \& Canaan, R.E., Simultaneous velocity measurements of both components of a two-phase flow using particle image velocimetry. International Journal of Multiphase Flow, 3, pp. 371-395, 1992. https://doi.org/10.1016/0301-9322(92)90023-a

[3] Westerweel, J., Fundamentals of digital particle image velocimetry. Measurement Science and Technology, 8, pp. 1379-1392, 1997. https://doi.org/10.1088/0957-0233/8/12/002

[4] Jensen, J., Flow measurements. Proceedings of ENCIT2004 XXVI, 4, pp. 400-419, 2004.

[5] Chen, R.C. \& Fan, L.S., Particle image velocimetry for characterizing the flow structure in three dimensional gas-liquid-solid fluidized beds. Chemical Engineering Science, 47, pp. 3615-3622, 1992. https://doi.org/10.1016/0009-2509(92)85077-o

[6] Birvalski, M., Tummers, M.J., Delfos, R. \& Henkes, R.A.W.M., PIV measurements of waves and turbulence in stratified horizontal two-phase pipe flow. International Journal of Multiphase Flow, 62, pp. 161-173, 2014. https://doi.org/10.1016/j.ijmultiphaseflow.2014.03.001

[7] Ayati, A.A., Kolaas, J., Jensen, A. \& Johnson, G.W., Combined simultaneous two-phase PIV and interface elevation measurements in stratified gas/liquid pipe flow. International Journal of Multiphase Flow, 74, pp. 45-58, 2015. https://doi.org/10.1016/j.ijmultiphaseflow.2015.03.024

[8] Kumara, W.A.S., Halvorsen, B.M. \& Melaaen, M.C., Single-beam gamma densitometry measurements of oil-water flow in horizontal and slightly inclined pipes. International Journal of Multiphase Flow, 36, pp. 467-480, 2010. https://doi.org/10.1016/j.ijmultiphaseflow.2010.02.003

[9] Olive, N.R., Zhang H.O., Wang O., Redus C.L. \& Brill J.P., Experimental study of low liquid loading gas-liquid flow in near-horizontal pipes. Journal Energy Resources Technology, 125, pp. 294-298, 2003. https://doi.org/10.1115/1.1625394

[10] Sutharshan, B., Kawaji, M. \& Ousaka, A., Measurement of circumferential and axial liquid film velocities in horizontal annular flow. International Journal of Multiphase Flow, 21, pp. 193-206, 1995. https://doi.org/10.1016/0301-9322(94)00070-z

[11] Newton, C.H. \& Behnia, M., A numerical model of stratified wavy gas-liquid pipe flows. Chemical Engineering Science, 56, pp. 6851-6861, 2001. https://doi.org/10.1016/s0009-2509(01)00322-0

[12] Meknassi, F., Benkirane, R., Liné, A. \& Masbernat, L., Numerical modeling of wavy stratified two-phase flow in pipes. Chemical Engineering Science, 55, pp. 4681-4697, 2000. https://doi.org/10.1016/s0009-2509(00)00070-1

[13] Valle, A., Multiphase pipeline flows in hydrocarbon recovery. Multiphase Science and Technology, 10, pp. 1-139, 1998.

https://doi.org/10.1615/multscientechn.v10.i1.10 